\title{
3D-Printed Portable Robotic Mobile Microscope for Remote Diagnosis of Global Health Diseases
}

\author{
Jaime García-Villena ${ }^{1}{ }^{\circledR}$, Jose Emilio Torres ${ }^{1}$, Carlos Aguilar ${ }^{1}{ }^{\circledR}$, Lin Lin ${ }^{1,2}{ }^{\circledR}$, David Bermejo-Peláez ${ }^{1}{ }^{1}$, \\ Elena Dacal ${ }^{1}$, Adriana Mousa ${ }^{1}$, María del Pilar Ortega ${ }^{1}$, Alvaro Martínez ${ }^{1}$, Alexander Vladimirov ${ }^{1}$, \\ Daniel Cuadrado ${ }^{1}$, María Postigo ${ }^{1}$, Jaume Ordi ${ }^{3}{ }^{\circ}$, Quique Bassat ${ }^{3,4,5,6,7} \mathbb{C}$, Javier Salamanca ${ }^{8}$, \\ Jose Luis Rodriguez-Peralto ${ }^{8}$, María Linares ${ }^{9,10}{ }^{(0)}$, Juan Enrique Ortuño ${ }^{2,11}$, María Jesús Ledesma-Carbayo ${ }^{2,11}$, \\ Andres Santos ${ }^{2,11}$ and Miguel Luengo-Oroz ${ }^{1, *}$
}

check for updates

Citation: García-Villena, J.; Torres, J.E.; Aguilar, C.; Lin, L.;

Bermejo-Peláez, D.; Dacal, E.; Mousa,

A.; Ortega, M.d.P.; Martínez, A.;

Vladimirov, A.; et al. 3D-Printed

Portable Robotic Mobile Microscope for Remote Diagnosis of Global Health Diseases. Electronics 2021, 10, 2408. https://doi.org/10.3390/ electronics10192408

Academic Editor: Manuel F. Silva

Received: 23 July 2021

Accepted: 23 September 2021

Published: 2 October 2021

Publisher's Note: MDPI stays neutral with regard to jurisdictional claims in published maps and institutional affiliations.

Copyright: (c) 2021 by the authors. Licensee MDPI, Basel, Switzerland. This article is an open access article distributed under the terms and conditions of the Creative Commons Attribution (CC BY) license (https:// creativecommons.org/licenses/by/ $4.0 /)$.
1 Spotlab, 28040 Madrid, Spain; jaime.garcia@spotlab.org (J.G.-V.); jetorresm@gmail.com (J.E.T.); carlos@spotlab.org (C.A.); lin@spotlab.org (L.L.); david@spotlab.org (D.B.-P.); elena@spotlab.org (E.D.); adrianamousa@spotlab.org (A.M.); pilar.ortega.arevalo@gmail.com (M.d.P.O.); alvaro@spotlab.org (A.M.); alex@spotlab.org (A.V.); danielcuadrado@spotlab.org (D.C.); maria@spotlab.org (M.P.)

2 Biomedical Image Technology Lab, Universidad Politécnica de Madrid, 28040 Madrid, Spain; juanen@die.upm.es (J.E.O.); mledesma@die.upm.es (M.J.L.-C.); andres@die.upm.es (A.S.)

3 ISGlobal, Hospital Clínic-Universitat de Barcelona, 08036 Barcelona, Spain; jaume.ordi@isglobal.org (J.O.); quique.bassat@isglobal.org (Q.B.)

4 Centro de Investigação em Saúde de Manhiça (CISM), Maputo 1929, Mozambique

5 ICREA, Pg. Lluís Companys 23, 08010 Barcelona, Spain

6 Pediatrics Department, Hospital Sant Joan de Déu, Universitat de Barcelona, 08034 Barcelona, Spain

7 Consorcio de Investigación Biomédica en Red de Epidemiología y Salud Pública (CIBERESP), 28029 Madrid, Spain

8 Department of Pathology, Hospital Universitario 12 de Octubre, 28041 Madrid, Spain; fsalam01@ucm.es (J.S.); jrperalto@salud.madrid.org (J.L.R.-P.)

9 Biochemistry and Molecular Biology Department, Pharmacy School, Universidad Complutense de Madrid, 28040 Madrid, Spain; mlinares@ucm.es

10 Hematology Department, Hospital 12 de Octubre, 28041 Madrid, Spain

11 Consorcio de Investigación Biomédica en Red de Bioingeniería, Biomateriales y Nanomedicina (CIBER-BBN), 28029 Madrid, Spain

* Correspondence: miguel@spotlab.org

Abstract: Microscopy plays a crucial role in the diagnosis of numerous diseases. However, the need for trained microscopists and pathologists, the complexity of pathology, and the accessibility and affordability of the technology can hinder the provision of rapid and high-quality diagnoses and healthcare. In this work, we present an affordable, 3D-printed, portable, robotic, mobile-based slide scanning microscope. The proposed device is composed of electronic, mechanical, and optical modules operated via smartphone with a control app. The device is connected and fully integrated with a telemedicine web platform, where digitized microscopy images can be remotely visualized and analyzed. The robotic scanner, which has approximately 1- $\mu \mathrm{m}$ resolution, has been evaluated in two clinical scenarios with histology and stool samples. The results showed sufficient image quality for performing a proper diagnosis in all cases under study.

Keywords: robotic microscope scanner; mobile-based; 3D printed; telemedicine

\section{Introduction}

Microscopy plays a crucial role in the evaluation and diagnosis required by many medical disciplines, including microbiology, parasitology, or pathology. Even though other types of microscope, e.g., transmission electron microscope and fluorescent microscope, are widely available in the market, brightfield microscopy remains the gold standard for image analysis and the diagnosis of many medical specialities. 
The technology used in optical microscopes has remained the same for the last decades. Most pathologists and microbiologists continue to use the same technology they were using fifty years ago and the workflows and protocols to follow are almost the same. The main difference is that the population has increased, and therefore the number of samples has increased along with the complexity of the diagnostic criteria. A serious limitation in low- and middle-income countries is the number of specialists, and the time they have to perform in-situ diagnosis is limited [1].

The increasing need for trained microscopists, their increasing workload, and the accessibility and affordability of the technology can represent insurmountable constraints to deliver high quality and rapid healthcare attention in resource-constrained settings. However, new low-cost portable digital microscopes that leverage ever-improving quality and affordability of manufacturing processes; communications, mobile phones, and sensors, may be the way forward to point of care (PoC) diagnosis in remote locations, avoiding misleading results and delays. Thus, systems that could digitize microscope slides in a rapid, simple, and cheap manner could help bypass the dearth of microscopes, and more importantly, the scarcity of trained microscopists, as well as allow for the more specialized or even automated (artificial intelligence based) evaluation of the slides requiring microscopic evaluation.

It was seventy years ago when the concept of portable microscopy was firstly conceived by the hand of McArthur [2], guiding the portable microscopy industry path for several decades with many iterations of portable microscopes commercialized [2,3]. With this approach, remote areas where high-risk patients reside could be reached, but there was still the extreme need for highly trained field microscopists. In 1985, the first digital microscope was invented by Hirox Co Ltd., a Japanese lens company [4]. In the same decade, with the advances of moldable plastic technology and computer design in the late 1980s, low-cost portable microscopes were conceived [5]. It was not until the end of the first decade of the twenty-first century when an explosion within this field occurred. With the collective technological advances of LEDs [6], smartphones, sensors, and communications, together with the expiration in 2009 of the FDM 3D printing technique patent and the RepRap Project [7], a new concept of portable, low-cost, mobile-based digital microscopes was born [8-11].

The penetration rate of the smartphone's market is expected to cover approximately $80 \%$ of the world's population by 2023 [12]. In addition, smartphone technology is advancing by leaps and bounds, incorporating new improvements in terms of sensors, software, electronics, and connectivity, thereby growing the computing power exponentially and reducing its cost every year. Thus, smartphones used together with networking and cloud SaaS (software as a service) solutions open the door to the possibility for effective and rapid digitalization, allowing for not only remote diagnosis, but also slide archives, quality control, opinion sharing, and importantly, training purposes.

As a result of this widespread use of smartphones and their capabilities, mobile health diagnostics solutions are becoming a game-changing technology that could offer primary attention in low resource settings delivering PoC diagnostics, therefore decentralizing, democratizing, and digitizing medical diagnostic care. Moreover, the use of smartphones combined with 3D printing technology allows for extending the smartphone capabilities to additional digitization applications.

Several studies proposed low-cost microscopes built with 3D printing and mobile technology for low-resource areas [5,10,13-17]. For instance, Switz et al. presented a mobile phone microscope that achieved a resolution of smaller than $5 \mu \mathrm{m}$ and a field of view of $10 \mathrm{~mm}^{2}$. They demonstrated its use in the identification of blood cells in blood smears and soil-transmitted helminth eggs in stool samples [10]. Skandarajah evaluated CellScope scanner, which uses a $20 \times$ objective lens, for oral cancer screening [16]. Vasiman et al. reviewed existing mobile phone and handheld microscopes and their application in malaria and neglected tropical diseases, finding that some of them have high sensitivity and specificity compared with conventional microscopes [5]. Even so, these solutions yield 
a set of images from different single fields from the sample, which in some cases are not enough to render a proper examination of the sample. As an example, pathologists have to see the whole slide to determine if the patient is healthy or the slide contains some malignant cells. In order to address this problem, whole-slide scanners are used.

Currently, commercial slide scanning technology that uses precise sub-micron motor stages to capture image tiles at a magnification and stitch them together entails a cost in the order of tens of thousands dollars [18-20]. An alternative is to use more affordable hardware with less precise position information and more sophisticated computer vision methods to stitch the slide together [21-23]. Li et al. presented Octopi, a scanner that is capable of automated slide scanning and multi-modal imaging. Instead of using nanometer precision motors, they used low-cost lead screw linear actuators. The device could be used for malaria and tuberculosis diagnostics and digital pathology [24]. Similarly, Collins et al. presented OpenFlexure, a 3D printed microscope scanner using low-cost stepper motors [11]. Nevertheless, implementation of those systems remains a difficult prospect for many institutions, especially those with stakeholders unfamiliar with these technologies.

Based on the previous considerations, this paper presents an affordable, 3D-printed, portable, robotic, mobile-based microscope scanner with an interactive augmented reality environment, connected to a telemicroscopy platform. We present the use of the system in different clinical applications, such as pathology and infectious diseases. We first (1) present all components (mechanics, optics, and mobile and cloud features) of the proposed robotic microscope; (2) summarize the performance assessment of the device and its application in two clinical scenarios; and (3) discuss its utility and possible future work lines derived from its use.

\section{Robotic Mobile-Based Microscope Scanner}

\subsection{System Architecture}

The system (Figure 1) is the result of the expansion of the capabilities of a smartphone, by leveraging the infrastructures that the device itself supplies. Namely, the camera for image capture, the Bluetooth module for communicating nearby devices in an abstract manner, and the WiFi and broadband cellular network modem for connectivity with remote systems via the internet.

A

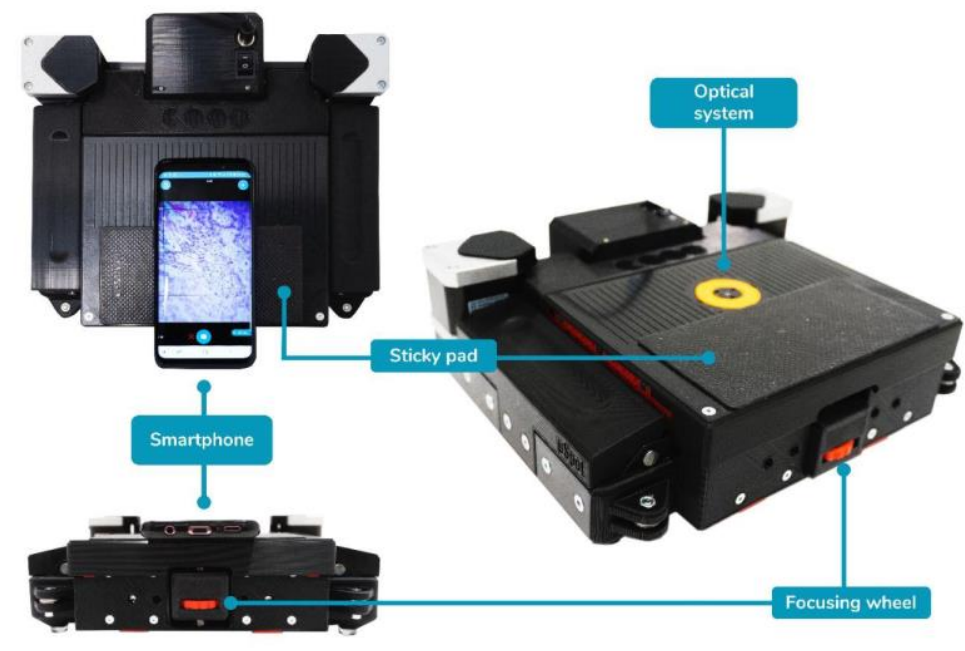

B

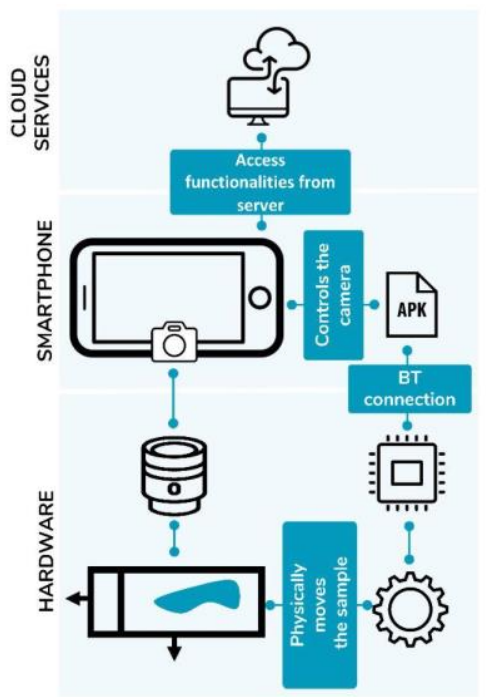

Figure 1. (A) Overview of the design of the proposed device. The phone is placed so it is aligned with the optics. Focusing wheel is used to move the vertical axis of the system for optical focus. (B) Interaction between the 3 main components of the device, including the hardware, the smartphone and cloud services. 
The smartphone is used in combination with an electromechanical device that implements the physical features needed in a microscopy scanner. Such electromechanical device is comprised of a mechanical cartesian XY stage powered by stepper motors that mounts a carriage capable of holding standard $78 \times 25 \mathrm{~mm}^{2}$ sample glasses. An electronics module comprising a power stage equipped with stepper drives, an ATMega4809 Microcontroller, and an ESP32 based SoC; and a miniaturized optic system designed to provide a usable digital image of a microscopy sample when coupled with the camera optics.

Higher level functionalities such as user interface, machine routines, asset organization, and client-side interaction with the servers are implemented on the App. Servers provide the infrastructure for tele-medicine features as well as any computationally heavy task.

\subsection{Mechanical Structure}

The mechanical stage of the proposed device has been developed with aim to produce a compact, cost-effective design, enabled by the production of $3 \mathrm{D}$ printed parts. This manufacturing method has been selected because, compared to other methods such as moulding, it is very flexible. It allows for rapid iteration on the designing phase, and on demand and distributed production, which is optimal to reduce $\mathrm{CO} 2$ footprint due to transportation.

Regarding compactness, the target size has been selected in such a way that the device can fit in a regular size backpack. A maximum boundary box of $250 \times 250 \times 150 \mathrm{~mm}^{3}$ was taken into consideration, for the design not to exceed in any of these dimensions. The design targets a low production cost that could only be achieved by minimizing the customization of parts, i.e., composing the assembled product with the most customer off-the-shelf parts as possible; preferably standard.

The custom needs of the design have been addressed by features in 3D printed parts which become the nodes that connect the hardware commercial off-the-shelf parts of the design and provide the special features that the requisites mandate. The whole design has been optimized for this manufacturing technique and organized in functionally distinct modules that, while not completely interchangeable nor easily disassembled by the user, allowed for decoupled development through iterations with different versions during development. The machine used for 3D printing was a Raise3D Pro 3D printer and the filaments used were Raise 3D Premium PLA Filament for all the parts except for the ring that encloses the optical system, which was 3D printed with Recreus Filaflex.

Every mechanical component (see Figure 2) is mounted onto the Main Frame, directly or indirectly, effectively making it a chassis. The frame structural composition stems from the union of two pairs of aluminium profiles forming a rectangle. Instead of using off-theshelf corner brackets or gussets, 3D printed corner parts have been used, which provide support for other modules, or actively participate in the other modules' functionalities, such as mounting the stepper motors, the $Y$-axis rods, and holding pulleys necessary for transmission in place. With the objective of minimizing the moving parts in the design, the actuation of such axes is laid out in a H-bot configuration. This allows for having the two stepper motors fixed to the chassis, reducing the number of moving parts.

In order to achieve focusing capabilities, a third vertical axis is needed in order to place the sample at the proper distance from the optics. While traditional microscopes move the sample stage towards the optics, our device moves the optics, along with the phone, closer to the sample by descending onto it. The vertical axis consists of a sliding platform anchored to the main rail, with vertical 3D printed linear rails to allow for vertical movement. Because friction between 3D printed parts is undesirable, small plastic spheres are used as rolling elements for these vertical rails. In order to actuate the linear motion, the front panel has a short spindle attached, whose nut is accessible from the front. The nut can be spun by the user using their thumb, causing a vertical movement on the spindle. 


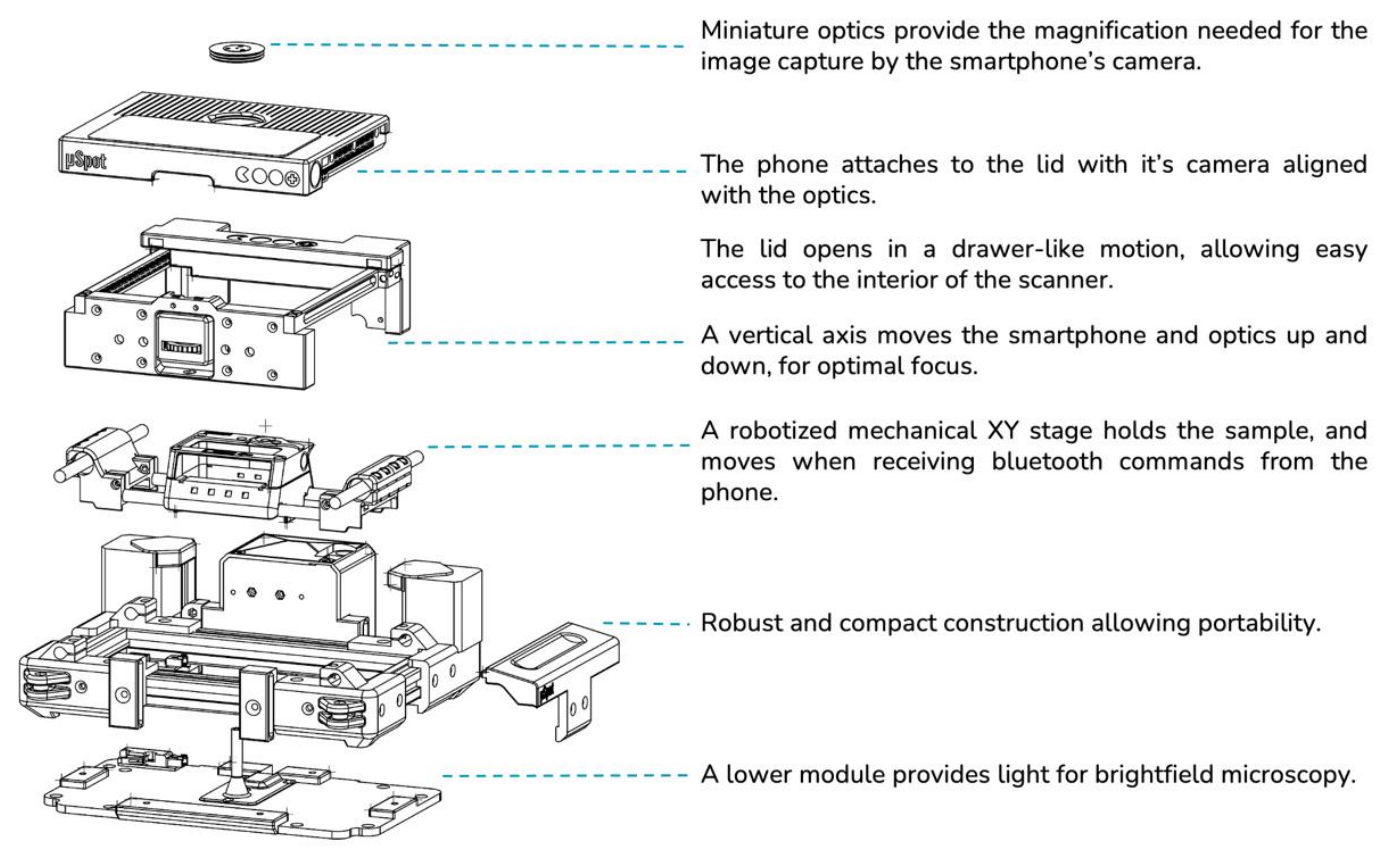

Figure 2. Exploded view of the proposed microscope scanner.

Mounted onto the cartesian stage's carriage is a magnetically attached tray designed to hold the sample glass. The tray has flat fixings akin to the ones present in a classic microscope stage, designed to not stand above the glass, avoiding collisions with the optics.

The mechanical stage is enclosed on its top side by an upper lid, that additionally hosts the optics module and provides means of holding the smartphone in place while device operation takes place. The holding means being a sticky pad, commercially used to attach smartphones onto car dashboards. The lid is mounted onto sliding rails using the same 3D printed rails used on the vertical axis, allowing to uncover the mechanical stage by sliding the lid to the front out of the way, allowing to place the sample onto the scan tray.

The optics have been designed with miniaturization as a priority. In order to reduce the optical path in length as much as possible, miniature lenses have been used, resulting in a "disk-like" design. The optics mount has an exterior ring, 3D printed in flexible material, designed to fit through a hole in the upper lid. The flexible coupling makes it easy to replace the optics in case different magnifications are needed, and it also provides a damping mechanism in the event of the optics crashing against the sample upon focusing, as many commercially available microscope objectives have.

\section{Electronic Layout and Firmware}

The device's electromechanical architecture shares the responsibility of operations between two microcontrollers: a mechanical microcontroller widely used on homemade $\mathrm{CNC}$ machining, and a Bluetooth enabled coordination microcontroller running upper-level Bluetooth communication firmware, responsible for communicating with the smartphone app and making upper level decisions based on the state of the machine. Additionally, means for actuating the axes are put in place, as well as illumination functionalities. Each axis is actuated by a stepper motor, equipped with a stepper driver, and an endstop. The endstops and the stepper drivers are connected to the mechanical microcontroller, the steppers being controlled via STEP/DIR signal pairs. A PWM line connects the mechanical microcontroller to an LED driver, which connects to a $3 \mathrm{~W}$ white light LED diode. The coordination microcontroller communicates the mechanical microcontroller via RX/TX serial lines, at 115,200 baud. The coordination microcontroller receives commands from and sends responses to the smartphone via bluetooth, and if concluded by some basic decision making, sends low-level movement commands to the mechanical microcontroller 
via the serial connection. The mechanical microcontroller reports back low-level status, e.g., pin states and axis position.

The mechanical microcontroller is an ATMega4809 running a CNC router firmware. The firmware implements a GCODE command interpreter, with step signal control for stepper drivers and endstop detection, governed by the states of a state machine. The firmware interprets GCODE commands received from the serial connection and coordinates the step signals to perform the requested movement. The state machine provides a readiness level that establishes a conservative approach to movement, to minimize the risk of mechanical damage. (e.g., no movement is performed unless the position of the stepper motors is known by the firmware via homing).

The coordination microcontroller is an ESP32 processor encapsulated within a NiNaW102 module, which provides antennas for WiFi and Bluetooth capabilities. The coordination microcontroller runs a firmware that connects to the mechanical microcontroller via serial connection and tracks its state. It establishes a Bluetooth connection with the app, acting as intermediary between the mechanical stage and the smartphone. Additionally, it provides construction information upon request, such as maximum and minimum coordinates, the characteristics of the lenses available in the device, as well as the illumination options. This information is later used to present magnification information to the user, as well as correct positioning.

Despite being carried over Bluetooth, communications with the smartphone are encoded with a REST API format for the requests, and a JSON format for the responses, to take advantage of the multiple smartphone app libraries available for parsing JSONformatted strings.

The motors in charge of making the movement effective are bipolar stepper motors. The chosen motors are of the NEMA 17 family, which are over dimensioned in load but that allows for a steady, cold operation. Moreover, material costs are driven down as these motors have been widely used in 3D printing for the last decade, which resulted in more availability for sourcing. The motors are driven by Pololu's A4988 stepper drivers, powered at $12 \mathrm{~V}$, and with their STEP/DIR pin pairs connected to the mechanical microcontroller's GPIOs. The motors are paired with 3D printed custom-made pulleys, and driven on $1 / 16$ microstep configuration they yield a mathematical XY resolution of $5.5 \mu \mathrm{m}$.

\subsection{Optics}

In order to make the image taken by the smartphone uniform among smartphones, the optics have been designed to be infinity-corrected. That way, as we will discuss later, it is possible to estimate the total magnification of the image projected onto the sensor, when the focal length of the camera lens is known. In order to reduce component costs, the optics consist of two customer off-the-shelf lenses, chosen from commercial catalogs (e.g., Edmund Optics and Thorlabs) and evaluated via simulation to provide the best compromise between field of view size and resolution on the center.

As a reference for simulations, the camera of a Samsung S9 was considered, which reportedly provides a camera with a $4.3 \mathrm{~mm}$ focal length lens, and a $4032 \times 3024$ pixel sensor, measuring $5648 \times 4236 \mathrm{~mm}^{2}$ in physical size. Simulations showed a total magnification of $2 \times$ (understanding this magnification as the relation of sizes between the object and the image projected onto the sensor), a numerical aperture of 0.13 , and a working distance of $0.92 \mathrm{~mm}$. Upon comparing these results with the ones obtained in a standard microscope, the resolution of images offered by this lens would be equivalent to a $10 \times$ microscope objective. Digital zoom is calculated and applied so that the field of view of digitized images is equivalent to the one obtained by a microscope objective of $40 \times$ magnification, with an eyepiece of field number 20, for a horizontal FOV of $0.5 \mathrm{~mm}$.

In order to make brightfield microscopy effective, the sample is backlighted by the aforementioned $3 \mathrm{~W}$ LED. The LED is enclosed in the lower lid of the main frame, and a tube directs the light up to the very bottom of the sample glass. In order to increase the 
numerical aperture of the lighting and prevent lens flare, a light-diffusing sheet is glued to the tip of the tube.

\subsection{Mobile Control App Features}

The frontend of the device is presented to the user through a smartphone application, which provides the user interface and provides the upper-level automated operation routines, such as automatic scanning. The minimum requirement for the smartphone is that the operating system is over Android 6 and the camera pixel resolution is greater than $1440 \times 1080$, after the digital zoom is applied. In cases of the resolution of the camera being lower than this requirement, the fidelity of the image will be affected by pixel interpolation algorithms.

\subsubsection{Augmented Reality Environment on Specimen}

The application is designed to orient the user throughout the exploration of the sample, through an augmented reality (AR) environment projected onto the display of the sample. For that, it keeps track of the position in which the carriage is placed, as well as the area of the sample that is visible on the screen at any given time.

Since API 21, Android has provided reports on a series of physical properties of the camera modules, such as those described above. Those properties, along with the focal length of the additional lens that the device provides, and the digital zoom selected by the use, can be used to calculate an estimate of the area that is visible on the phone screen. Moreover, the application monitors the position of the carriage within the device, and therefore the position of the center of the visible field of the sample is known at all times.

That information is used to provide the user with a layout containing useful information that is understandable and meaningful to the professionals in the field, namely a virtual grid displayed on the screen, on top of the sample display. The size of the cells of such a grid are calculated using the data described above, in such a way that each cell encloses an area of the displayed sample that would correspond to a $0.1 \times 0.1 \mathrm{~mm}^{2}$ area onto the real sample. Furthermore, the grid cells are numbered in such a way that the user is always oriented in relation to their position on the sample, as well as the actual size of the displayed objects.

This layout element, being responsive to the area of the sample displayed on the screen, effectively provides an AR environment that enhances the exploration of the sample, providing metrics that enhance the orientation of the user upon exploring the sample.

\subsubsection{Mechanical Control}

The application constitutes the upper command level of the device as a whole. That includes control over the mechanics as well as the illumination. By conception, the device attempts to provide a familiar and user-friendly interface and operation to the user, and the mechanical control is designed according to such a concept.

The application effectively transforms the three well-known gestures (panning, flinging, and pinching) into movements of the microscope, as well as zooming onto the sample. In the case of panning and flinging, the movement of the finger across the screen is translated into micrometric movements of the carriage, that correspond to the movement of the finger scaled for magnification, in such a way that the finger is always pointing to the same zone on the sample. Pinching is used for zoom, translating the movement of the fingers into digital zoom, adjusting the magnification at the user's will.

The aforementioned virtual grid is updated in real time upon user interaction, in such a way that it always corresponds to the area of the sample that is displayed on the screen.

In this way, the application brings the smartphone control over the mechanics of the device, as well as collecting imaging information from the examined sample, via the camera feed. This effectively creates a closed control loop that can be used to implement fully automated routines such as a scan procedure, in which an area of the sample is swept across, step by step, making evenly spaced photos distributed in a grid. The distance 
between each photo's coordinates is calculated using the sample area visible to the camera, in such a way that an overlap exists between the photos, allowing for a later stitching into a big photo map of the whole area.

The device can execute this task unattended, which allows for non-specialized operators to perform the scanning process. The scan photos can be uploaded onto a cloud server for specialized personnel to analyze.

\subsection{Remote Telemedicine Platform}

All acquired images using the mobile app are transferred via the mobile network to an Amazon Simple Storage Service (Amazon S3, Seattle, WA, USA) bucket, which is encrypted with the industry-standard AES-256 encryption algorithm. In addition, data security in transit is ensured through enforcing HTTPS (Secure HTTPS) through TLS (Transport Layer Security).

The images can be visualized through a remote telemedicine platform entirely developed within SpotLab, where images are presented in an easy-to-use dashboard that allows their management, sharing, analysis and reporting [25]. The remote platform, which is accessible from all desktops, laptops, tablets, and mobile phones, translates standard clinical diagnostic protocols into digital tasks which are adapted to each case under study. Figure 3 illustrates a screenshot of the telemedicine platform.

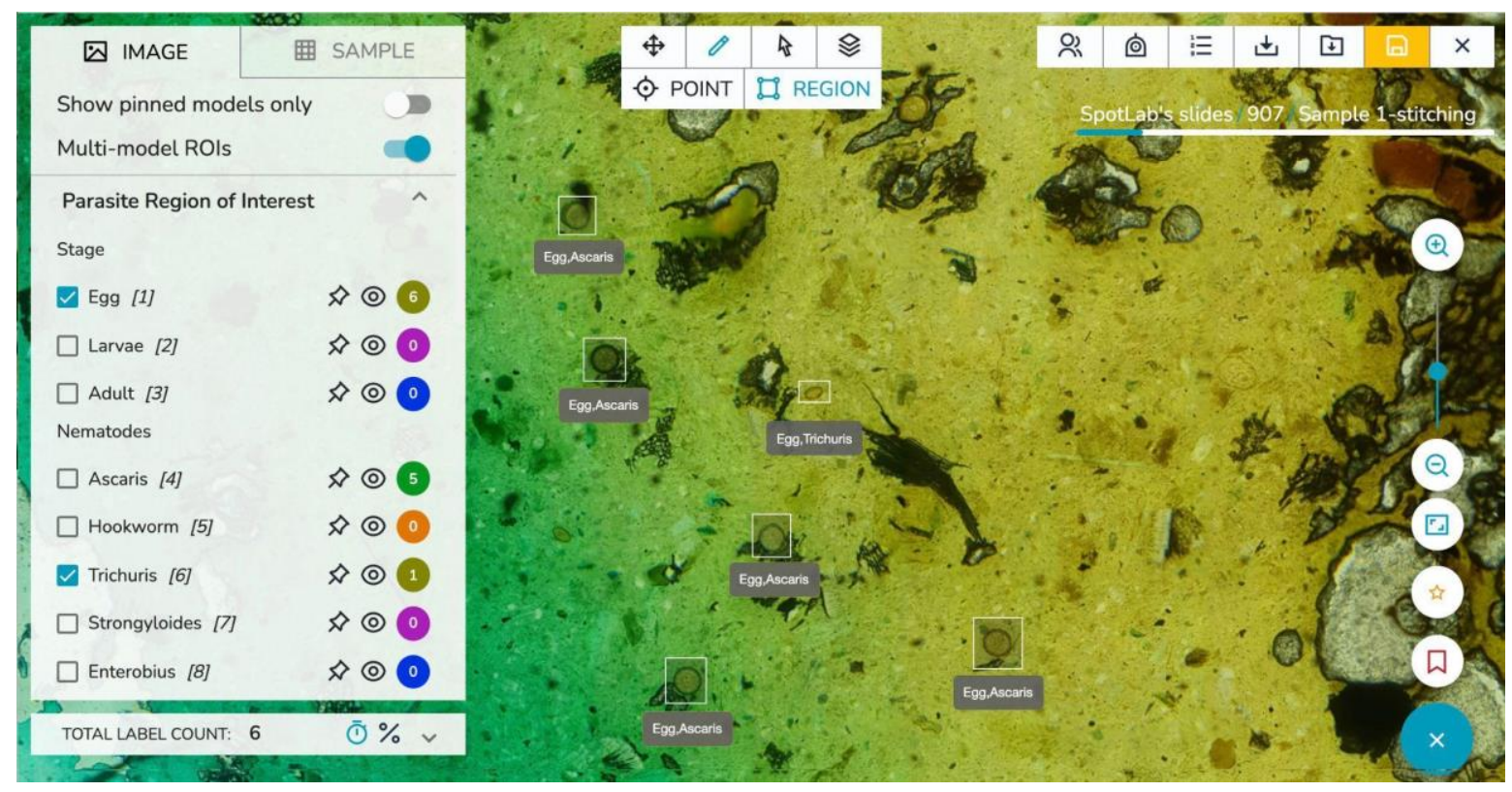

Figure 3. Screenshot of the remote telemedicine platform (developed by the authors) which allows users to navigate and zoom the image, perform annotations, quantify relevant objects and provide overall diagnostic assessments. The sample shown in this example is a Kato-Katz preparation.

As previously mentioned, a scan routine is implemented to acquire the necessary captures to produce whole slide images so that once all individual images are uploaded, an image processing pipeline is automatically triggered to stitch and generate the final whole slide image. This processing pipeline includes several steps such as vignetting correction without reference or background image, integrated distortion correction, pairwise registration between adjacent scan images and fusion using Voronoi cells and Laplacian blending. A detailed description of the stitching algorithm as well as its performance are presented elsewhere [26]. This stitching algorithm is deployed in Amazon Web Services using a docker container. Resulting stitched images are also uploaded to the Amazon S3 container and can be visualized through the telemedicine platform. 


\section{Performance Assessment}

In order to evaluate the performance of the optical system, the slanted edge test [27] was used to determine the optical resolution of the optical system. We imaged a slanted edge from a R1L3S5P resolution target (Thorlabs, Inc., Newton, NJ, USA) (Figure 4A,B), computed the edge spread function, derived it, and obtained the modulation transfer function (MTF) (Figure 4C). The MTF describes the ability of an optical system to transfer the object contrast to the acquired image as a function of frequency and can be used to determine the system resolution. Table 1 reports the spatial resolution of the system quantified as the spatial frequency at different levels of the MTF. Note that spatial frequencies between $10 \%$ and $20 \%$ MTF (MTF10, MTF20) correspond to the classic vanishing resolution, while MTF50 ensures resolution where objects can be imaged with sharpness and detail. As derived from Table 1, the proposed system is able to distinguish objects as small as approximately 1 micrometer $(\mu \mathrm{m})$.

A

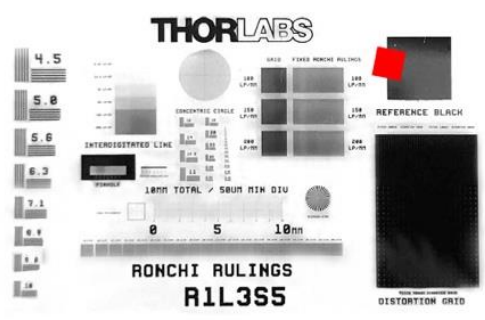

B

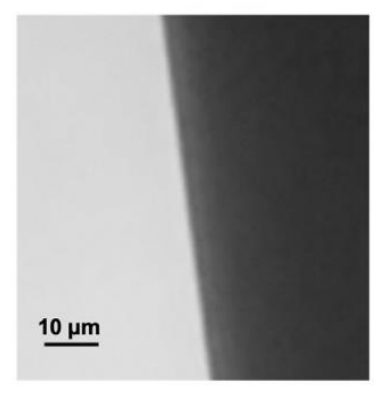

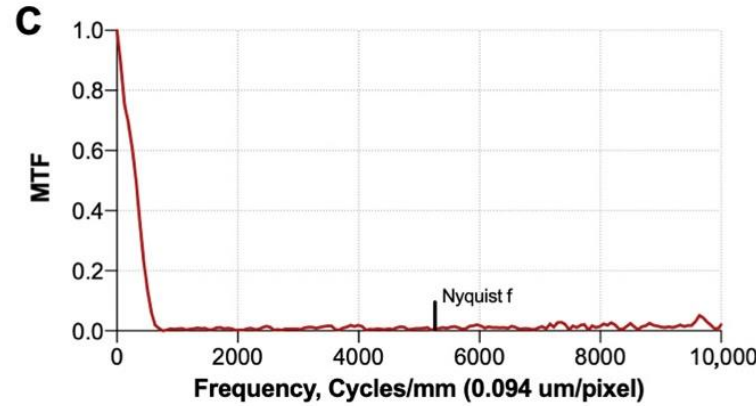

Figure 4. (A) R1L3S5P resolution test target, (B) Slanted test image (red rectangle from resolution target), (C) Modulation transfer function (MTF).

Table 1. Spatial resolution metrics for different frequency cut-offs of the Modulation Transfer Function derived from a slanted edge test.

\begin{tabular}{cc}
\hline MTF Cutoff (\%) & Spatial Resolution \\
\hline 10 & $577 \mathrm{lp} / \mathrm{mm}$ \\
20 & $474 \mathrm{lp} / \mathrm{mm}$ \\
50 & $330 \mathrm{lp} / \mathrm{mm}$ \\
\hline
\end{tabular}

To compare the quality of the images obtained through our proposed robotic microscope to the one of the images obtained with a conventional microscope, we have digitized the same sample with both the proposed device and a standard microscope (Leica DM-200, $40 \times$ objective, field number 22). The image corresponding to the conventional microscope was obtained by coupling a smartphone to the microscope's eyepiece. Both images were captured with the same device (Samsung S9). As shown in Figure 5, the quality of the images is similar and all objects of interest that can be visualized using the conventional microscope can also be correctly identified using our proposed device.

Regarding the performance of the scan procedure and the image processing pipeline, it should be noted that a typical $20 \times 20$ grid scan at a magnification of $40 \times$ that covers a field of view of $7.2 \times 7.2 \mathrm{~mm}^{2}$ can be obtained in under $5 \mathrm{~min}$. Figure 6 shows an example of a stitched scan composed by $20 \times 20$ independent acquisitions. 

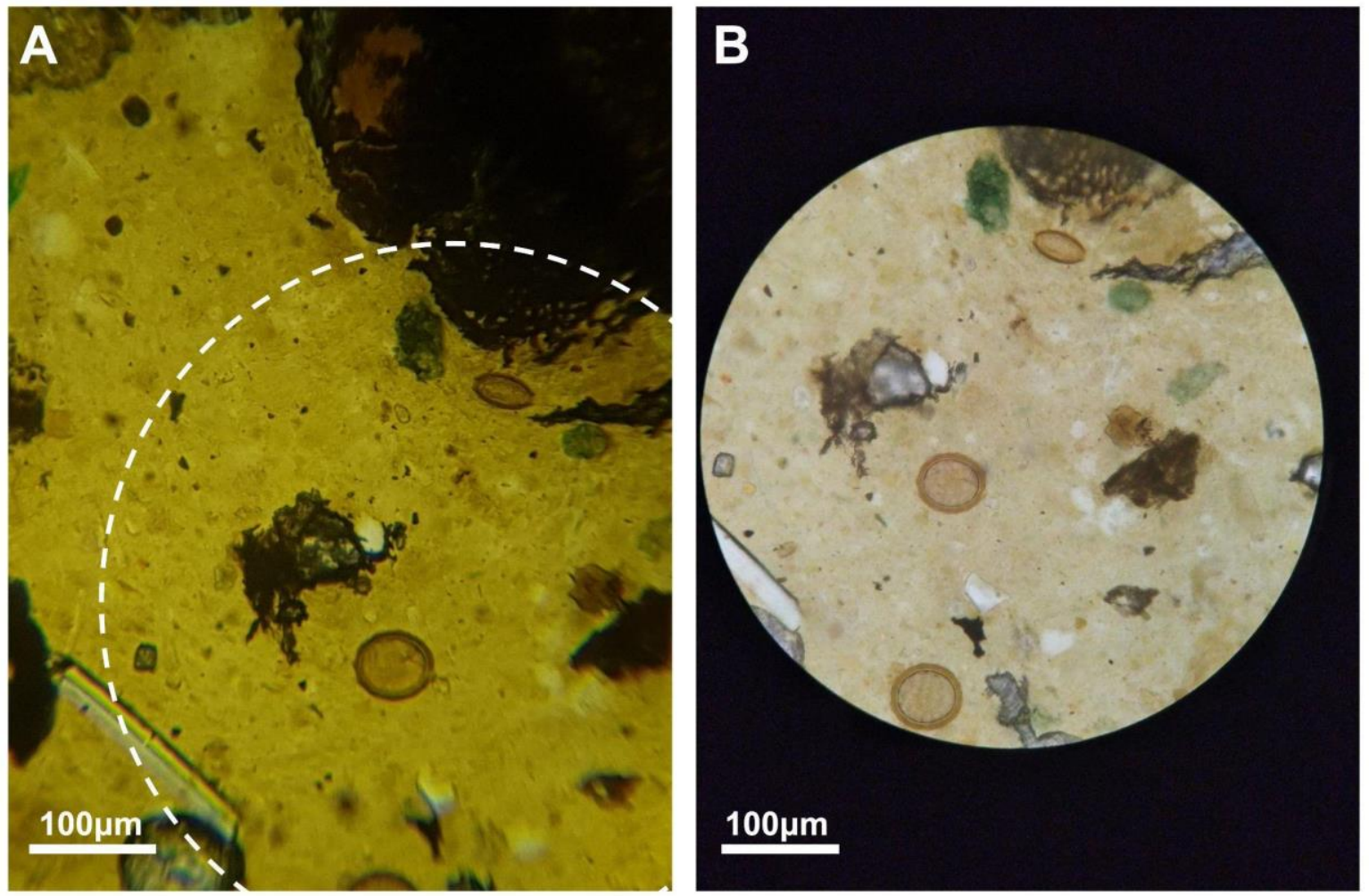

Figure 5. Comparison of images obtained from the same sample (Kato-Katz preparation) using (A) our proposed robotic microscope and (B) a conventional microscope (Leica DM-2000, 40× objective, field number 22). Both images were digitized using a Samsung S9. Note that the dashed line in panel A represents the digitized field of view presented in panel B.

A

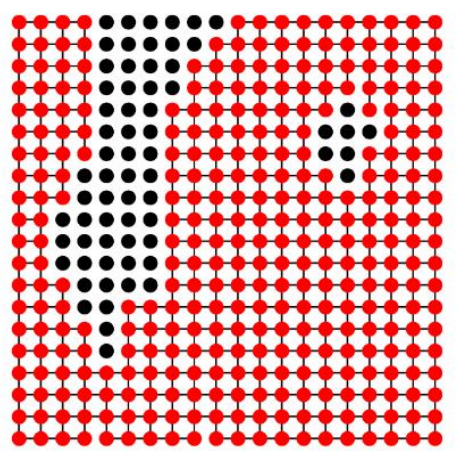

B

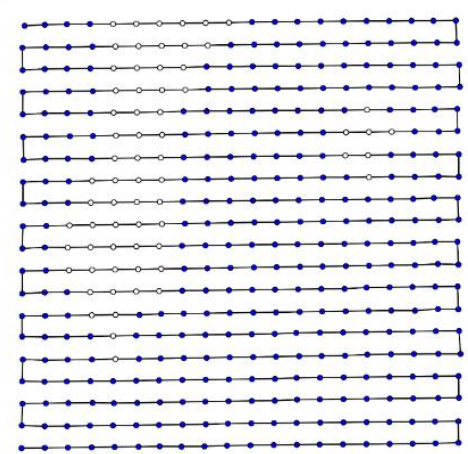

C

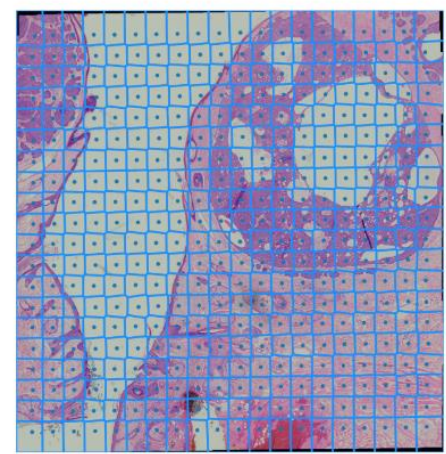

Figure 6. (A) Array of $20 \times 20$ independent images. Red dots represent successful registration between adjacent images, black dots represent unregistered images (background areas without significant features). (B) Scanner path. Unregistered images (white dots) are interpolated from the grid of registered images (blue dots). (C) Voronoi diagram representing the final stitched result.

The uploading time for such a scan is about $7 \mathrm{~min}$ on a $2 \mathrm{Mb} / \mathrm{s}$ connection, and the stitching process is performed in the cloud within $8 \mathrm{~min}$. For a field of view of $7.2 \times 7.2 \mathrm{~mm}^{2}$, an image of approximately $15,000 \times 15,000$ pixels is created, with a resulting pixel size of $0.48 \mu \mathrm{m} / \mathrm{mm}$ and a size of around $150 \mathrm{MB}$. These specifications fall within the typical range of commercial scanners [20]. 


\section{Clinical Applications}

4.1. Histopathology

Histopathology is the diagnosis and study of diseases through the study of morphological characteristics on tissues or cells under microscopy. It is an essential field for diagnosing all those diseases that directly affect the cell structure or tissue, with an extended use and linkage to the field of oncology. In 2020, 19.3 million new cancer cases were estimated to occur, and this number is expected to rise to 28.4 million by 2040 [28]. However, not all hospitals have anatomical pathology departments or have enough pathologists. In 2018, The Royal College of Pathologists reported that only 3\% of anatomical pathology departments in the UK had enough pathologists to meet clinical demand [29]. China, US, Africa as a continent, and many other countries have also reported a significant shortage of pathologists [30-32]. Cost-effective devices that process and digitize histopathological samples in an automatic manner, and which are connected to a telemedicine platform, may alleviate these limitations, particularly if they can be linked to automated interpretation software.

Digital pathology focuses on the acquisition, digitalization, and management of specimen slides through computer-based technologies, improving the quality of the analysis. Digital pathology includes several steps, such as scanning the slide, stitching images to create whole slide images (WSI), visualizing and sharing it with other pathologists in a computer-based environment.

Whole slide imaging can make a great difference in pathology. The white paper from the Digital Pathology Association described the use cases of WSI, including slide archiving, remote consultation and telepathology, in-line scanning, tumor board, education, and research [23]. In addition, it allows pathology departments to continue working remotely in case of public health emergencies [33]. Furthermore, artificial intelligence-based image analysis can be further developed to reduce the workload of pathologists [34,35].

To evaluate the usability of the proposed device for diagnosing diseases through histopathology, we digitized a total of nine different hematoxylin and eosin (HE) stained slides of tissues. We investigated a total of eight lesions, including colon tubular adenoma, lung adenocarcinoma, acinar prostate adenocarcinoma, high-grade breast ductal invasive carcinoma, endometrial polyp, basocellular carcinoma, epidermoid carcinoma, and seborrheic keratoses, as well as healthy thyroid gland tissue. As already mentioned, the samples were scanned at $40 \times$ magnification by using the proposed robotic scanner. Three different pathologists visually evaluated the digitized images of all samples, and all could perform a proper diagnosis of all samples, supporting the concept that digitized images generated by the proposed device demonstrate sufficient quality for performing histopathological diagnosis. However, it should be noted that pathologists indicated that some images presented some limitations, such as limited resolution and non-uniform focus along the entire image, which created difficulties for the identification of some specific structures such as cell nucleoli. An example of an automated stitched scan image of a $20 \times 20$ grid of captures of a colon tubular adenocarcinoma histology sample is shown in Figure 7. 

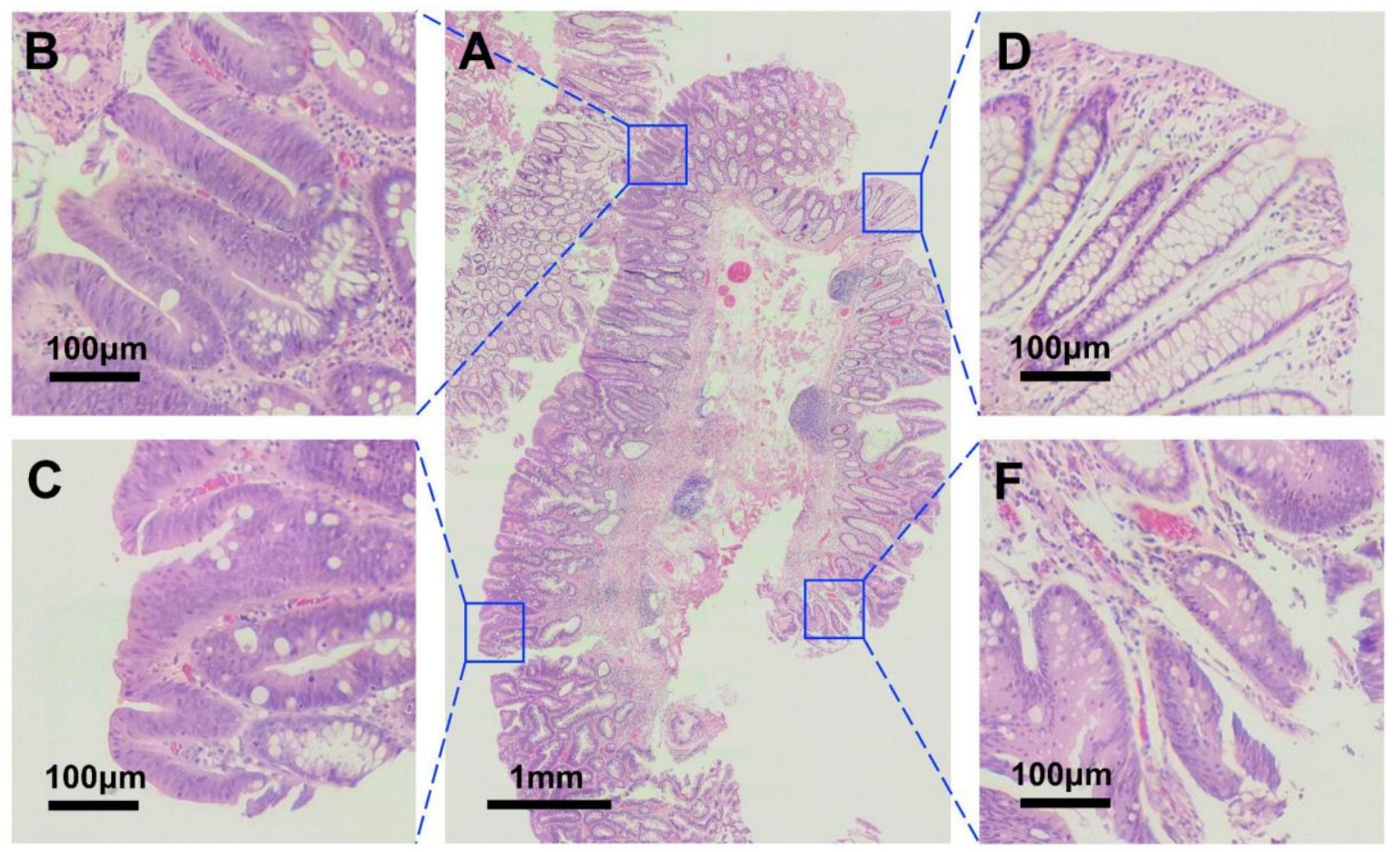

Figure 7. (A) Stitched scan image $(20 \times 20$ grid $)$ of a colon tubular adenocarcinoma histology sample. (B-F) High magnification images showing different structures of interest.

\subsection{Infectious Diseases}

Infectious diseases are caused by a wide range of microorganisms, including bacteria, viruses, fungi, and parasites, and remain the most important cause of morbidity and mortality in low-income countries. Many infectious diseases, including malaria, tuberculosis, or a variety of bacterial infections (treponemas causing syphilis, pneumococcus and other bacteria causing pneumonia or meningitis etc.), can benefit from microscopic diagnosis. Beyond these, another group of infections which has vastly been neglected in the past are the so-called neglected tropical diseases (NTDs). These diseases are found in the so-called $10 / 90$ gap, where less than $10 \%$ of global funding for research is spent on diseases that afflict more than $90 \%$ of the world's population [36,37]. Within NTDs, the most prevalent group relates to soil-transmitted helminths $(\mathrm{STH})$, an infectious disease that affects approximately 1.5 billion people, representing $24 \%$ of the world's population, principally in the low-income countries in tropical and subtropical areas [38].

The WHO roadmap to combat NTDs by 2021-2030 has recently been published [39]. In the case of STHs, it is proposed that $96 \%$ of countries should have eliminated STHs as a public health problem by 2030. In addition to using a mass drug administration (MDA) strategy to control these diseases on a regular basis, the Kato-Katz (KK) technique is recommended as a diagnostic method. This microscopy-based technique is inexpensive and allows quantification of eggs by classifying individuals into three grades of low, moderate or heavy infection intensity. Patients may be asymptomatic or, if severely infected, may have symptoms, such as diarrhoea, abdominal pain, and malnutrition, which undermine the growth of children $[37,40]$.

The main drawback of the KK technique is that it requires a quick reading of the sample because helminth eggs -especially those of hookworms- tend to disappear as time goes by [41]. This fact could be solved by digitizing the sample, as this would allow for a later reading to be made without the need for the sample to deteriorate.

Several methods for the digitization of slides of stool samples as well as its automatic analysis assisted by artificial intelligence algorithms have been already proposed [42-44]. 
However, it should be noted that all previously proposed approaches require manual intervention for digitizing the samples and were not based on an automatic scanner procedure.

To demonstrate the usefulness of the proposed robotic scanner for digitizing stool samples to perform a subsequent diagnosis of STHs infections, we have digitized nine Kato-Katz slides from nine different subjects. All images were remotely analysed through the telemedicine web platform by tagging those regions where an Ascaris spp. or Trichuris spp. eggs were detected. Additionally, and for comparative purposes, all samples were also analyzed using a conventional microscopy procedure. Intensity of infection was quantified using both approaches (digitized images and conventional microscopy) by using a four-point scale ("-" 0; "+" 1-9 eggs; "++" 10-99 eggs and "+++" more than 100 eggs). Comparison between readings is shown in Figure 8A. Weighted kappa was calculated to assess the degree of agreement between the two techniques, and as depicted in the table, almost perfect agreement was obtained (kappa scores of 0.816 and 1 for quantification of Trichuris spp. and Ascaris spp. respectively).

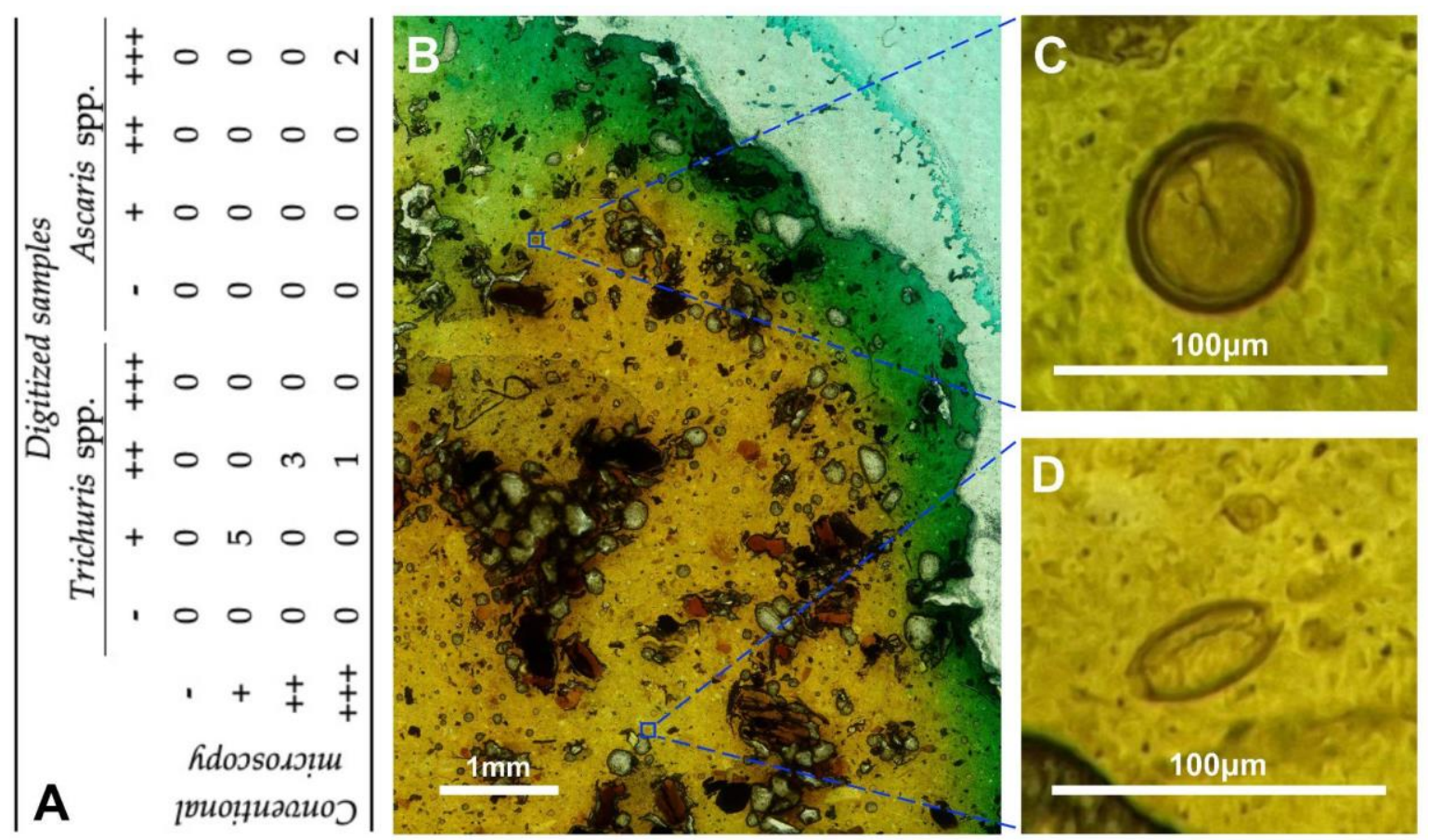

Figure 8. (A): Confusion matrix between intensity of infection performed by conventional microscopy and based on digitized samples. (B): Automated $20 \times 20$ grid scan from a Kato-Katz slide of a stool sample. (C): High magnification image showing Ascaris spp. egg. (D): Magnification image showing Trichuris spp. egg.

Figure $8 \mathrm{~B}-\mathrm{D}$ shows a stitched tile scan obtained from a $20 \times 20$ grid of captures of a stool sample co-infected by Trichuris spp. and Ascaris spp. eggs. This figure shows the potential of the proposed microscope to correctly digitize images with enough image quality to be able to perform an identification of different STH parasites.

\section{Discussion and Conclusions}

At the present time, there is an urgent need, especially in low resource settings, for quality and low-cost point of care microscopy scanner diagnosis devices. In this work, we have presented an affordable, 3D-printed, portable, robotic, mobile-based microscope scanner, fully integrated with a telemedicine platform. It presents a combination of a simple $3 \mathrm{D}$ printed automatic stage with the increased computing and image-capturing power of smartphones, which allows for a completely automated, cost-effective, and mobile-based solution to microscope sample scanning. 
The device can be built in non-specialized manufacturing environments on demand and made of less than $1.5 \mathrm{~kg}$ of 3D printed plastic parts, common hardware parts (such as normalized bolts and nuts, aluminum profiles, and smooth rods), off-the-shelf optics, and widely adopted electromechanic components, making it at least as affordable as other entry level scanners. The resulting weight of the device is around $3 \mathrm{~kg}$.

Its compact design allows for physical portability, while its conceptualization around the smartphone hardware allows for connectivity, portability, and ease of use. Both the phone and device are combined in a compact package that is convenient for its operation in remote areas, by non-specialized personnel. Additionally, the proposed robotic microscope scanner is also connected to a telemedicine platform enabling remote diagnosis and analysis of digitized samples.

The proposed device has been assessed in two clinical scenarios, including histopathology (different tissue pathologies) and infectious diseases (soil transmitted helminthiasis). The results support its adequate usability and sufficient performance quality to allow diagnosis. Therefore, the quality of the digitized microscopy images generated by the proposed robotic microscope appear suitable for distinguishing and identifying all structures of interest for performing a proper diagnosis.

Future lines of work may benefit from the fact that the smartphone is responsible for capturing and ultimately storing the images as the media files used for later analysis, e.g., enabling the use of edge-detecting image algorithms. The use of AI algorithms to digest the camera feed allows for different in-situ automated and semi-automated routines, such as locking on to parasites or features detected by the AI algorithm when exploring the sample, or auto-searching specific features in a sample, making photos of areas of interest in a completely automated way. Two more example routines that could improve the user experience through the information received via the camera feed can be implemented, namely (1) a further enhancement of the augmented reality environment where features of the sample detected by artificial intelligence algorithms can be highlighted, and (2) an autofocus routine, with a future version of the device that provides a motorized Z-axis, to be operated according to focus-detecting measurements obtained from the camera feed. It should be noted that AI algorithms may also be integrated in the telemedicine platform for the automatic analysis of uploaded images, thus assisting experts in the diagnosis of different pathologies. Additionally, future lines of work will consider the validation of the proposed robotic microscope for the digitalization of other microscopic images and diagnosis of different pathologies. An cost analysis of the proposed device and the potential impact in low-resource settings will also be considered.

The development of cost-effective solutions affordable for physicians is key in the democratization of diagnosis and for the general improvement of the quality of care offered. The proposed robotic microscope digitization device aims to provide access to democratized and decentralized diagnosis, contributing to the achievement of universal health care.

Author Contributions: Technology, J.G.-V., J.E.T., C.A., L.L., D.B.-P., E.D., A.M. (Adriana Mousa), M.d.P.O., A.M. (Alvaro Martínez), A.V., D.C., M.P., J.E.O., M.J.L.-C., A.S., and M.L.-O.; clinical validation, E.D., M.P., J.O., Q.B., J.S., J.L.R.-P., M.L., and M.L.-O.; funding acquisition, M.P. and M.L.-O.; writing—original draft preparation, J.G.-V., C.A., L.L., D.B.-P., E.D., M.L., and M.L.-O.; writing-review and editing, J.G.-V., C.A., L.L., D.B.-P., E.D., M.L., and M.L.-O. All authors have read and agreed to the published version of the manuscript.

Funding: This work has been partially funded by projects CDTI NEOTEC SNEO-20171197, EU SME Instrument Phase 2-881062, and by IND2019/TIC-17167 (Comunidad de Madrid).

Institutional Review Board Statement: The study was conducted according to the guidelines of the Declaration of Helsinki. Ethical approval for acquiring anonymized samples used in this study was obtained from the Kenya Medical Research Institute (KEMRI) Ethics Review Committee (SERU 3873) and from the Hospital 12 de Octubre Ethics Review Committee (20/430).

Data Availability Statement: All relevant data are within the manuscript. 
Acknowledgments: We thank Dra. Stella Kepha from KEMRI (Kenya) for providing the stool samples used for evaluation.

Conflicts of Interest: JGV, JET, CA, LL, DBP, ED, AMo, AMa, AV, DC, MP, QB, ML, MJLC, AS and MLO hold shares or phantom shares of Spotlab. The rest of the authors declare no conflict of interest.

\section{References}

1. World Health Organization. World Health Statistics 2020: Monitoring Health for the Sdgs, Sustainable Development Goals; World Health Organization: Geneva, Switzerland, 2020; p. viii. 77p.

2. McArthur, J. Advances in the design of the inverted prismatic microscope. J. R. Microsc. Soc. 1945, 65, 8-16. [CrossRef]

3. Dunning, K.; Stothard, J.R. From the mcarthur to the millennium health microscope (MHM): Future developments in microscope miniaturization for international health. Microsc. Today 2007, 15, 18-21. [CrossRef]

4. Hirox-3D Digital Microscope-Hirox Europe. Available online: https://hirox-europe.com/ (accessed on 25 March 2021).

5. Vasiman, A.; Stothard, J.R.; Bogoch, I.I. Mobile Phone Devices and Handheld Microscopes as Diagnostic Platforms for Malaria and Neglected Tropical Diseases (NTDs) in Low-Resource Settings: A Systematic Review, Historical Perspective and Future Outlook. Adv. Parasitol. 2019, 103, 151-173. [CrossRef] [PubMed]

6. Wessels, J.T.; Pliquett, U.; Wouters, F.S. Light-emitting diodes in modern microscopy-from David to Goliath? Cytom. Part A 2012, 81, 188-197. [CrossRef] [PubMed]

7. RepRap-RepRap. Available online: https://reprap.org/wiki/RepRap (accessed on 22 April 2021).

8. Breslauer, D.N.; Maamari, R.N.; Switz, N.; Lam, W.A.; Fletcher, D.A. Mobile phone based clinical microscopy for global health applications. PLoS ONE 2009, 4, e6320. [CrossRef] [PubMed]

9. Hassan, S.E.-D.H.; Okoued, S.I.; Mudathir, M.A.; Malik, E.M. Testing the sensitivity and specificity of the fluorescence microscope $\left(\right.$ Cyscope $^{\circledR}$ ) for malaria diagnosis. Malar. J. 2010, 9, 88. [CrossRef] [PubMed]

10. Switz, N.; D'Ambrosio, M.V.; Fletcher, D.A. Low-cost mobile phone microscopy with a reversed mobile phone camera lens. PLoS ONE 2014, 9, e95330. [CrossRef] [PubMed]

11. Collins, J.T.; Knapper, J.; Stirling, J.; Mduda, J.; Mkindi, C.; Mayagaya, V.; Mwakajinga, G.A.; Nyakyi, P.T.; Sanga, V.L.; Carbery, D.; et al. Robotic microscopy for everyone: The OpenFlexure microscope. Biomed. Opt. Express 2020, 11, 2447-2460. [CrossRef]

12. Smartphone Users 2021 I Statista. Available online: https:/ / www.statista.com/statistics/330695/number-of-smartphone-usersworldwide/ (accessed on 15 July 2021).

13. Agbana, T.E.; Diehl, J.-C.; Van Pul, F.; Khan, S.; Patlan, V.; Verhaegen, M.; Vdovin, G. Imaging \& identification of malaria parasites using cellphone microscope with a ball lens. PLoS ONE 2018, 13, e0205020. [CrossRef]

14. Ozcan, A. Mobile phones democratize and cultivate next-generation imaging, diagnostics and measurement tools. Lab Chip 2014, 14, 3187-3194. [CrossRef]

15. Mudanyali, O.; Tseng, D.; Oh, C.; Isikman, S.O.; Sencan, I.; Bishara, W.; Oztoprak, C.; Seo, S.; Khademhosseini, B.; Ozcan, A. Compact, light-weight and cost-effective microscope based on lensless incoherent holography for telemedicine applications. Lab Chip 2010, 10, 1417-1428. [CrossRef] [PubMed]

16. Skandarajah, A.; Sunny, S.P.; Gurpur, P.; Reber, C.D.; D'Ambrosio, M.V.; Raghavan, N.; James, B.L.; Ramanjinappa, R.D.; Suresh, A.; Kandasarma, U.; et al. Mobile microscopy as a screening tool for oral cancer in India: A pilot study. PLoS ONE 2017, 12, e0188440. [CrossRef]

17. Coulibaly, J.T.; Ouattara, M.; D'Ambrosio, M.V.; Fletcher, D.A.; Keiser, J.; Utzinger, J.; N'Goran, E.K.; Andrews, J.R.; Bogoch, I.I. Accuracy of Mobile Phone and Handheld Light Microscopy for the Diagnosis of Schistosomiasis and Intestinal Protozoa Infections in Côte d'Ivoire. PLoS Negl. Trop. Dis. 2016, 10, e0004768. [CrossRef] [PubMed]

18. Isse, K.; Lesniak, A.; Grama, K.; Roysam, B.; Minervini, M.I.; Demetris, A.J. Digital transplantation pathology: Combining whole slide imaging, multiplex staining and automated image analysis. Arab. Archaeol. Epigr. 2011, 12, 27-37. [CrossRef]

19. Chalfoun, J.; Majurski, M.; Blattner, T.; Bhadriraju, K.; Keyrouz, W.; Bajcsy, P.; Brady, M. MIST: Accurate and Scalable Microscopy Image Stitching Tool with Stage Modeling and Error Minimization. Sci. Rep. 2017, 7, 1-10. [CrossRef] [PubMed]

20. Pantanowitz, L.; Farahani, N.; Parwani, A. Whole slide imaging in pathology: Advantages, limitations, and emerging perspectives. Pathol. Lab. Med. Int. 2015, 7, 23-33. [CrossRef]

21. Beckstead, J.A.; Dawson, R.; Feineigle, P.A.; Gilbertson, J.J.; Hauser, C.; McVaugh, T.; Palmieri, F.; Sholehvar, D.; Wetzel, A. High-throughput high-resolution microscopic slide digitization for pathology. Adv. Biomed. Clin. Diagn. Syst. 2003, 4958, 149-160. [CrossRef]

22. Montalto, M.C.; McKay, R.R.; Filkins, R.J. Autofocus methods of whole slide imaging systems and the introduction of a second-generation independent dual sensor scanning method. J. Pathol. Inform. 2011, 2, 44. [CrossRef]

23. Zarella, M.; Bowman, D.; Aeffner, F.; Farahani, N.; Xthona, A.; Absar, S.F.; Parwani, A.; Bui, M.; Hartman, D.J. A practical guide to whole slide imaging: A white paper from the digital pathology association. Arch. Pathol. Lab. Med. 2019, 143, 222-234. [CrossRef] [PubMed]

24. Li, H.; Soto-Montoya, H.; Voisin, M.; Valenzuela, L.F.; Prakash, M. Octopi: Open configurable high-throughput imaging platform for infectious disease diagnosis in the field. BioRxiv 2019, preprint. [CrossRef] 
25. Dacal, E.; Bermejo-Peláez, D.; Lin, L.; Álamo, E.; Cuadrado, D.; Martínez, Á.; Mousa, A.; Postigo, M.; Soto, A.; Sukosd, E.; et al. Mobile microscopy and telemedicine platform assisted by deep learning for the quantification of Trichuris trichiura infection. PLoS Negl. Trop. Dis. 2021, 15, e0009677. [CrossRef] [PubMed]

26. Ortuño, J.E.; Lin, L.; Ortega, M.D.P.; Garcia-Villena, J.; Cuadrado, D.; Linares, M.; Santos, A.; Ledesma-Carbayo, M.J.; LuengoOroz, M. Stitching Methodology for Whole Slide Low-Cost Robotic Microscope Based on a Smartphone. In Proceedings of the 2020 IEEE 17th International Symposium on Biomedical Imaging (ISBI), Iowa City, IA, USA, 3-7 April 2020 ; pp. 503-507. [CrossRef]

27. Burns, P.D. Slanted-Edge MTF for Digital Camera and Scanner Analysis. In Proceedings of the PICS: Image Processing, Image Quality, Image Capture, System Conference, Portland, OR, USA, 26 March 2000.

28. Sung, H.; Ferlay, J.; Siegel, R.L.; Laversanne, M.; Soerjomataram, I.; Jemal, A.; Bray, F. Global cancer statistics 2020: Globocan estimates of incidence and mortality worldwide for 36 cancers in 185 countries. CA Cancer J. Clin. 2021, 71, 209-249. [CrossRef] [PubMed]

29. Histopathology Workforce Survey 2018. Available online: https://www.rcpath.org/profession/workforce-planning/ourworkforce-research/histopathology-workforce-survey-2018.html (accessed on 26 April 2021).

30. China Grapples with a Pathologist Shortage-Global HealthCare Insights Magazine. Available online: https://globalhealthi.com/ 2017/10/19/china-pathologist-shortage/ (accessed on 26 April 2021).

31. Mudenda, V.; Malyangu, E.; Sayed, S.; Fleming, K. Addressing the shortage of pathologists in Africa: Creation of a MMed Programme in Pathology in Zambia. Afr. J. Lab. Med. 2020, 9, 974. [CrossRef] [PubMed]

32. Metter, D.M.; Colgan, T.J.; Leung, S.T.; Timmons, C.F.; Park, J.Y. Trends in the US and canadian pathologist workforces from 2007 to 2017. JAMA Netw. Open 2019, 2, e194337. [CrossRef] [PubMed]

33. Hanna, M.G.; Reuter, V.E.; Ardon, O.; Kim, D.; Sirintrapun, S.J.; Schüffler, P.J.; Busam, K.J.; Sauter, J.L.; Brogi, E.; Tan, L.K.; et al. Validation of a digital pathology system including remote review during the COVID-19 pandemic. Mod. Pathol. 2020, 33, 2115-2127. [CrossRef]

34. Huang, Y.-N.; Peng, X.-C.; Ma, S.; Yu, H.; Jin, Y.-B.; Zheng, J.; Fu, G.-H. Development of Whole Slide Imaging on Smartphones and Evaluation With ThinPrep Cytology Test Samples: Follow-Up Study. JMIR mHealth uHealth 2018, 6, e82. [CrossRef]

35. Niazi, M.K.K.; Parwani, A.V.; Gurcan, M.N. Digital pathology and artificial intelligence. Lancet Oncol. 2019, 20 , e253-e261. [CrossRef]

36. Vanderslott, S. Moving from outsider to insider status through metrics: The inclusion of 'neglected tropical diseases' into the sustainable development goals. J. Hum. Dev. Capab. 2019, 20, 418-435. [CrossRef]

37. Global Forum for Health Research and World Heath Organization. The 10/90 (Ten Ninety) Report on Health Research 2003-2004; Global Forum for Health Research: Geneva, Switzerland, 2004.

38. Pullan, R.L.; Freeman, M.C.; Gething, P.; Brooker, S.J. Geographical inequalities in use of improved drinking water supply and sanitation across Sub-Saharan Africa: Mapping and spatial analysis of cross-sectional survey data. PLoS Med. 2014, 11, e1001626. [CrossRef]

39. WHO TEAM. Ending the Neglect to Attain the Sustainable Development Goals: A Road Map for Neglected Tropical Diseases 2021-2030; World Health Organization: Geneva, Switzerland, 2020; Available online: http:/ /www.who.int/neglected_diseases/WHONTDroadmap-2030/en/ (accessed on 18 September 2020).

40. Jourdan, P.M.; Lamberton, P.H.L.; Fenwick, A.; Addiss, D.G. Soil-transmitted helminth infections. Lancet 2018, 391, 252-265. [CrossRef]

41. Dacombe, R.; Crampin, A.; Floyd, S.; Randall, A.; Ndhlovu, R.; Bickle, Q.; Fine, P. Time delays between patient and laboratory selectively affect accuracy of helminth diagnosis. Trans. R. Soc. Trop. Med. Hyg. 2007, 101, 140-145. [CrossRef]

42. Holmström, O.; Linder, N.; Ngasala, B.; Mårtensson, A.; Linder, E.; Lundin, M.; Moilanen, H.; Suutala, A.; Diwan, V.; Lundin, J. Point-of-care mobile digital microscopy and deep learning for the detection of soil-transmitted helminths and Schistosoma haematobium. Glob. Health Action 2017, 10, 1337325. [CrossRef] [PubMed]

43. Yang, A.; Bakhtari, N.; Langdon-Embry, L.; Redwood, E.; Lapierre, S.G.; Rakotomanga, P.; Rafalimanantsoa, A.; Santos, J.D.D.; Vigan-Womas, I.; Knoblauch, A.M.; et al. Kankanet: An artificial neural network-based object detection smartphone application and mobile microscope as a point-of-care diagnostic aid for soil-transmitted helminthiases. PLoS Negl. Trop. Dis. 2019, 13, e0007577. [CrossRef] [PubMed]

44. Li, Q.; Li, S.; Liu, X.; He, Z.; Wang, T.; Xu, Y.; Guan, H.; Chen, R.; Qi, S.; Wang, F. FecalNet: Automated detection of visible components in human feces using deep learning. Med. Phys. 2020, 47, 4212-4222. [CrossRef] [PubMed] 